\title{
Reactive Oxygen Species and Mitochondrial $\mathrm{K}_{\mathrm{ATP}}$ Channels Mediate Helium-Induced Preconditioning Against Myocardial Infarction In Vivo
}

\author{
Paul S. Pagel, MD PhD, John G. Krolikowski, BS, Phillip F. Pratt Jr, PhD, Yon Hee Shim, \\ MD, Julien Amour, MD PhD, David C. Warltier, MD PhD, and Dorothee Weihrauch, DVM PhD \\ From the Department of Anesthesiology, the Medical College of Wisconsin and the Clement J. \\ Zablocki Veterans Affairs Medical Center, Milwaukee, Wisconsin.
}

\begin{abstract}
Objectives-Helium produces preconditioning by activating prosurvival kinases, but the roles of reactive oxygen species (ROS) or mitochondrial $\mathrm{K}_{\mathrm{ATP}}$ channels in this process are unknown. We tested the hypothesis that ROS and mitochondrial $\mathrm{K}_{\mathrm{ATP}}$ channels mediate helium-induced preconditioning in vivo.

Design-Randomized, prospective study.

Setting-University research laboratory.

Participants-Male New Zealand white rabbits.

Interventions-Rabbits ( $\mathrm{n}=64)$ were instrumented for measurement of systemic hemodynamics and subjected to a $30 \mathrm{~min}$ left anterior descending coronary artery (LAD) occlusion and $3 \mathrm{~h}$ reperfusion. In separate experimental groups, rabbits ( $\mathrm{n}=7$ or 8 per group) were randomly assigned to receive $0.9 \%$ saline (control) or three cycles of $70 \%$ helium-30\% oxygen administered for 5 min interspersed with $5 \mathrm{~min}$ of an air-oxygen mixture before LAD occlusion with or without the ROS scavengers $N$-acetylcysteine (NAC; $150 \mathrm{mg} / \mathrm{kg}$ ) or $N$-2-mercaptoproprionyl glycine (2-MPG; $75 \mathrm{mg} /$ $\mathrm{kg}$ ), or the mitochondrial $\mathrm{K}_{\mathrm{ATP}}$ antagonist 5-hydroxydecanoate (5-HD; $5 \mathrm{mg} / \mathrm{kg}$ ). Statistical analysis of data was performed with analysis of variance for repeated measures followed by Bonferroni's modification of Student's t test.
\end{abstract}

\begin{abstract}
Measurements and Main Results-Myocardial infarct size was determined using triphenyltetrazolium chloride staining and presented as a percentage of the left ventricular area at risk. Helium significantly $(\mathrm{P}<0.05)$ reduced infarct size $(23 \pm 4 \%$ of the area at risk; mean $\pm \mathrm{SD})$ compared with control (46 $\pm 3 \%)$. NAC, 2-MPG, and 5-HD did not affect irreversible ischemic injury when administered alone $(49 \pm 5,45 \pm 6$, and $45 \pm 3 \%)$, but these drugs blocked reductions in infarct size produced by helium $(45 \pm 4,45 \pm 2$, and $44 \pm 3 \%)$.
\end{abstract}

\footnotetext{
Submit all correspondence to: Paul S. Pagel MD PhD, Clement J. Zablocki Veterans Affairs Medical Center, Anesthesia Service, 5000 W. National Avenue, Milwaukee, Wisconsin 53295. Phone: (414) 384-2000, extension 42417; Facsimile: (414) 384-2939; Electronic mail:pspagel@mcw.edu.

Publisher's Disclaimer: This is a PDF file of an unedited manuscript that has been accepted for publication. As a service to our customers we are providing this early version of the manuscript. The manuscript will undergo copyediting, typesetting, and review of the resulting proof before it is published in its final citable form. Please note that during the production process errors may be discovered which could affect the content, and all legal disclaimers that apply to the journal pertain.

Preliminary data in this manuscript were presented in abstract form (Anesth Analg 2007;104:S-27) at the $81{ }^{\text {st }}$ Congress of the International Anesthesia Research Society, Orlando, Florida, March 2007.

The authors have no conflicts of interest pursuant to the current work.
} 
Conclusions-The results suggest that ROS and mitochondrial $\mathrm{K}_{\mathrm{ATP}}$ channels mediate heliuminduced preconditioning in vivo.

\section{Keywords}

myocardial ischemia; preconditioning; helium; reactive oxygen species; mitochondrial $\mathrm{K}_{\mathrm{ATP}}$ channels

Reperfusion after coronary artery occlusion produces large quantities of reactive oxygen species (ROS) that contribute substantially to myocardial injury ${ }^{1,2}$. In contrast, small amounts of ROS released from mitochondria during a brief episode of ischemia before prolonged coronary occlusion and reperfusion cause preconditioning 3,4 . Pretreatment with free radical scavengers abolished cardioprotection produced by ischemic preconditioning 5 . Volatile anesthetics also directly produce small quantities of $\operatorname{ROS}^{6,7}$ (most likely from mitochondrial electron transport chain complex $\mathrm{III}^{8}$ ) through activation of mitochondrial adenosine triphosphate-regulated potassium $\left(\mathrm{K}_{\mathrm{ATP}}\right)$ channels 9,10 . As observed during ischemic preconditioning, ROS generated by this form of pharmacologic preconditioning mediated reductions in myocardial infarct size produced by the volatile agent $6,7,10$. Brief, intermittent administration of helium before prolonged coronary artery occlusion and reperfusion was recently shown to protect myocardium against infarction by activating prosurvival signaling kinases [e.g., phosphotidylinositol-3-kinase (PI3K), extracellular signal-regulated kinases (Erk1/2), endothelial nitric oxide synthase (eNOS)], attenuating the detrimental actions of glycogen synthase kinase, and inhibiting mitochondrial transition in vivo ${ }^{11-13}$. Whether oxygen-derived free radical intermediates play a role in helium-induced preconditioning is unknown. Brief exposure to the anesthetic noble gas xenon produced cardioprotection by activating mitochondrial $\mathrm{K}_{\mathrm{ATP}}$ channels ${ }^{14}$, but the role of mitochondrial $\mathrm{K}_{\mathrm{ATP}}$ channels in cardioprotection by nonanesthetic noble gases remains undefined. Thus, the current investigation also tested the hypothesis that activation of mitochondrial $\mathrm{K}_{\mathrm{ATP}}$ channels mediates helium-induced preconditioning in rabbits.

\section{Methods}

All experimental procedures and protocols used in this investigation were reviewed and approved by the Animal Care and Use Committee of the Medical College of Wisconsin. Furthermore, all conformed to the Guiding Principles in the Care and Use of Animals of the American Physiologic Society and were in accordance with the Guide for the Care and Use of Laboratory Animals.

\section{Experimental Preparation}

Male New Zealand white rabbits weighing between 2.5 and $3.0 \mathrm{~kg}$ were anesthetized with intravenous sodium pentobarbital $(30 \mathrm{mg} / \mathrm{kg})$ as previously described ${ }^{11}$. Additional doses of pentobarbital were titrated as required to assure that pedal and palpebral reflexes were absent throughout the experiment. Briefly, a tracheostomy was performed through a midline incision, and each rabbit was ventilated with positive pressure using an air-oxygen mixture (fractional inspired oxygen concentration $=0.30$ ). Arterial blood gas tensions and acid-base status were maintained within a normal physiological range by adjusting the respiratory rate or tidal volume throughout the experiment. A pulse oximeter was placed on the right hind paw of each rabbit for measurement of continuous arterial oxygen saturation. Heparin-filled catheters were positioned in the right carotid artery and the left jugular vein for measurement of arterial blood pressure and fluid or drug administration, respectively. Maintenance fluids ( $0.9 \%$ saline; 15 $\mathrm{ml} \cdot \mathrm{kg}^{-1} \cdot \mathrm{min}^{-1}$ ) were continued for the duration of each experiment. A thoracotomy was performed at the left fourth intercostal space, and the heart was suspended in a pericardial cradle. A prominent branch of the left anterior descending coronary artery (LAD) was 
identified, and a silk ligature was placed around this vessel approximately halfway between the base and the apex for the production of coronary artery occlusion and reperfusion. Intravenous heparin $(500 \mathrm{U})$ was administered immediately before LAD occlusion. Coronary artery occlusion was verified by the presence of epicardial cyanosis and regional dyskinesia in the ischemic zone, and reperfusion was confirmed by observing an epicardial hyperemic response. Hemodynamics were continuously recorded on a polygraph throughout each experiment.

\section{Experimental Protocol}

The experimental design is illustrated in Figure 1. Baseline hemodynamics, arterial blood gas tensions, and arterial oxygen saturation were recorded $30 \mathrm{~min}$ after instrumentation was completed. All rabbits underwent a $30 \mathrm{~min}$ LAD occlusion followed by $3 \mathrm{~h}$ of reperfusion. In eight separate groups, rabbits ( $\mathrm{n}=7$ to 8 per group) were randomly assigned (Latin square design) to receive $0.9 \%$ saline (control) or three cycles of $70 \%$ helium-30\% oxygen administered for $5 \mathrm{~min}$ interspersed with $5 \mathrm{~min}$ of $70 \%$ nitrogen-30\% oxygen before coronary artery occlusion in the presence or absence of the ROS scavengers $\mathrm{N}$-acetylcysteine (NAC; $150 \mathrm{mg} / \mathrm{kg}$ ) or $N$-2-mercaptoproprionyl glycine (2-MPG; $75 \mathrm{mg} / \mathrm{kg}$ ), or the selective mitochondrial $\mathrm{K}_{\mathrm{ATP}}$ channel antagonist 5-hydroxydeconate (5-HD; $5 \mathrm{mg} / \mathrm{kg}$ ). NAC and 2MPG were dissolved in $0.9 \%$ saline and administered as intravenous infusions over 30 and 75 min, respectively. 5-HD was dissolved in $0.9 \%$ saline and administered intravenously $30 \mathrm{~min}$ before LAD occlusion. The doses of NAC, 2-MPG, and 5-HD used in the current investigation did not produce hemodynamic effects nor affect infarct size when administered alone in an identical rabbit model 7,10 . The doses of NAC, 2-MPG, and 5-HD also abolished isofluraneinduced production of ROS as detected by dihydroethidium staining independent of prolonged coronary artery occlusion and reperfusion in rabbits ${ }^{7,10}$. Taken together, these previous findings suggest that these particular antagonists are effective and selective in the doses that were used in the current investigation.

\section{Measurement of Myocardial Infarct Size}

Myocardial infarct size was measured as previously described ${ }^{15}$. Briefly, the LAD was reoccluded at the completion of each experiment and $3 \mathrm{ml}$ of patent blue dye was injected intravenously. The left ventricular area at risk for infarction was separated from surrounding normal areas (stained blue), and the two regions were incubated at $37^{\circ} \mathrm{C}$ for $20 \mathrm{~min}$ in $1 \%$ 2,3,5-triphenyltetrazolium chloride in $0.1 \mathrm{M}$ phosphate buffer adjusted to $\mathrm{pH}$ 7.4. Infarcted and noninfarcted myocardium within the area at risk were carefully separated and weighed after storage overnight in 10\% formaldehyde. Myocardial infarct size was expressed as a percentage of the area at risk. Rabbits that developed intractable ventricular fibrillation and those with an area at risk less than $15 \%$ of total left ventricular mass were excluded from subsequent analysis.

\section{Statistical Analysis}

Statistical analysis of data within and between groups was performed with multiple analysis of variance (ANOVA) for repeated measures followed by Bonferroni's modification of Student's $t$ test ${ }^{16}$. Changes were considered statistically significant when $\mathrm{P}<0.05$. All data are expressed as mean \pm standard deviation (SD).

\section{RESULTS}

Sixty-four rabbits were instrumented to obtain 59 successful infarct size experiments. Two rabbits were excluded because the left ventricular area at risk was less than $15 \%$ of the total left ventricular mass. Three rabbits were excluded because intractable ventricular fibrillation occurred during coronary artery occlusion. Arterial blood gas tensions were maintained within 
the physiologic range during administration of helium in all groups (data not shown). Arterial oxygen saturation remained at $100 \%$ during and after administration of helium with or without other drug interventions (data not shown). Baseline systemic hemodynamics were similar between groups (table 1), but mean arterial pressure was greater in rabbits randomized to receive $2-\mathrm{MPG}$ compared with $0.9 \%$ saline. Helium did not affect hemodynamics. Mean arterial pressure and rate-pressure product were greater in rabbits receiving NAC or 2-MPG alone before $\mathrm{LAD}$ occlusion compared with those treated with $0.9 \%$ saline. Brief coronary artery occlusion and reperfusion significantly $(\mathrm{P}<0.05)$ reduced rate-pressure product in all experimental groups. There were no differences in hemodynamics between groups during LAD occlusion and reperfusion. Body weight, left ventricular mass, area at risk weight, and the ratio of area at risk to left ventricular mass were similar between groups (table 2). Left ventricular weight and area at risk weight were less in rabbits randomized to receive NAC compared with $0.9 \%$ saline, but the ration of area at risk to left ventricular mass was similar between these groups. Brief, intermittent exposure to $70 \%$ helium before LAD occlusion reduced myocardial infarct size $(23 \pm 4 \%$ of the left ventricular area at risk) as compared with control rabbits (46 $\pm 3 \%$; figure 2). Administration of NAC, 2 -MPG, or 5-HD alone did not affect infarct size (49 $\pm 5,45 \pm 6$, and $45 \pm 3 \%$, respectively), but these drugs abolished helium-induced cardioprotection ( $45 \pm 4,44 \pm 2$, and $44 \pm 3 \%$, respectively).

\section{DISCUSSION}

The current results confirm our previous findings ${ }^{11-13}$ indicating that three cycles of 5 min $70 \%$ helium-30\% oxygen preconditioning interspersed with $5 \mathrm{~min}$ washout periods of an airoxygen mixture reduce myocardial necrosis after prolonged coronary artery occlusion and reperfusion. The results demonstrate for the first time that pretreatment with NAC or 2-MPG abolishes this helium-induced cardioprotection, suggesting that ROS mediate preconditioning by the nonanesthetic noble gas in vivo. The results further indicate that 5-HD pretreatment blocks reductions in myocardial infarct size produced by brief, intermittent administration of helium, implicating mitochondrial $\mathrm{K}_{\mathrm{ATP}}$ channels in this process as well. Weber et al demonstrated that mitochondrial $\mathrm{K}_{\mathrm{ATP}}$ channels mediated cardioprotection produced by the anesthetic inert gas xenon ${ }^{14}$, and the current results extend this observation to another noble gas that is devoid of anesthetic properties, even under extreme hyperbaric conditions ( $>200$ $\mathrm{atm})^{17}$. Collectively, the current and previous ${ }^{14}$ data with noble gases also lend support to the hypothesis that ROS are a ubiquitous feature in preconditioning phenomena in conjunction with mitochondrial $\mathrm{K}_{\mathrm{ATP}}$ channels. Pretreatment with low concentrations of ROS mimicked the beneficial effects of ischemic preconditioning 18 , and small quantities of ROS generated by mitochondria during brief periods of ischemia ${ }^{3,4}$ or after exposure to the mitochondrial $\mathrm{K}_{\mathrm{ATP}}$ channel agonist diazoxide ${ }^{19,20}$ have been shown to play a central role in ischemic and pharmacologic preconditioning, respectively. Evidence that mitochondrial $\mathrm{K}_{\mathrm{ATP}}$ activation mediates this ROS-induced cardioprotection was also provided by the observations that diazoxide enhances oxidation of the ROS probe Mitotracker® orange ${ }^{20}$ (Molecular Probes, Eugene, OR) and increases ROS production as measured using 2', 7'-dichlorofluorescein diacetate in rat ventricular myocytes or isolated hearts ${ }^{21}$. Pretreatment with 5-HD abolished ROS generation produced by the mitochondrial $\mathrm{K}_{\mathrm{ATP}}$ channel openers nicorandil and cromakalim in isolated rat hearts ${ }^{22}$. Similarly, the ROS scavengers NAC, 2-MPG, and Mn(III) tetrakis(4-benzoic acid)porphyrine chloride inhibited the cardioprotective effects of isoflurane in isolated 6 and intact rabbit hearts 7,10 . Scavengers of ROS also abolished the salutary actions of sevoflurane against ischemic damage in isolated guinea pig hearts ${ }^{23,24}$. Preconditioning by isoflurane directly increased production of superoxide anion $\left(\mathrm{O}_{2}^{-}\right)$as determined using dihydroethidium staining through a mitochondrial $\mathrm{K}_{\mathrm{ATP}}$ channel-mediated mechanism 8,10 . Thus, it has become abundantly clear that ROS and mitochondrial $\mathrm{K}_{\mathrm{ATP}}$ channels play complimentary roles during ischemic, pharmacologic, and anesthetic preconditioning, and the 
current results with helium suggest that ROS and mitochondrial $\mathrm{K}_{\text {ATP }}$ channels are also essential in preconditioning by the nonanesthetic noble gas.

The current results must be interpreted within the constraints of several potential limitations. Based on our previous experiments conducted with volatile anesthetics $7,8,10$, it appears highly likely that $\mathrm{O}_{2}{ }^{-}$generated from the electron transport chain though mitochondrial $\mathrm{K}_{\text {ATP }}$ channel opening may also responsible for the observed results with helium. The sulfhydryl-containing glutathione precursor NAC produces antioxidant effects by enhancing glutathione synthesis, acting as a substrate for glutathione peroxydase, and facilitating metabolism of hydrogen peroxide $\left(\mathrm{H}_{2} \mathrm{O}_{2}\right)$ by univalent reduction of $\mathrm{O}_{2}^{-}$via preservation of intracellular reduced glutathione concentration ${ }^{25}$. Large amounts of superoxide dismutase are contained within mitochondria, and this enzyme is primarily responsible for the chemical conversion of $\mathrm{O}_{2}{ }^{-}$to $\mathrm{H}_{2} \mathrm{O}_{2}$ and water. The subsequent reduction of $\mathrm{H}_{2} \mathrm{O}_{2}$ is catalyzed by glutathione peroxydase to which reduced glutathione serves as an electron donor during the reaction. Thus, the current observation that NAC abolishes helium-induced preconditioning indirectly infers that $\mathrm{O}_{2}{ }^{-}$or one of its immediate derivatives is responsible for this cardioprotective effect. 2-MPG also donates sulfhydryl groups to glutathione peroxydase and may be more mitochondria-specific than $\mathrm{NAC}^{26-28}$. These observations also indirectly suggest that $\mathrm{O}_{2}^{-}$derived from mitochondria or another oxygen-derived free radical intermediate produced by $\mathrm{O}_{2}{ }^{-}$metabolism is involved in cardioprotection by helium. Nevertheless, such conclusions must be qualified because we did not specifically determine the identity or define the source of the ROS involved in helium-induced preconditioning in the current investigation. It is also unclear based on the current results whether mitochondrial $\mathrm{K}_{\mathrm{ATP}}$ opening acts as a trigger or end-effector for preconditioning by helium through ROS generation. Our laboratory is currently examining this hypothesis.

In addition to previously described limitations, the current results must be interpreted within the constraints of several other potential shortcomings. The duration of administration of NAC, 2-MPG, and 5-HD were heterogeneous, and these pharmacokinetic factors may have influenced the results. Plasma concentrations of NAC, 2-MPG, and 5-HD were also not determined nor were dose-response relationships to these drugs performed. Myocardial infarct size is determined primarily by the size of the area at risk and the extent of coronary collateral perfusion. The area at risk expressed as a percentage of total left ventricular mass was similar between groups in the current investigation, and coronary collateral blood flow has been shown to be minimal in rabbits ${ }^{29}$. Thus, differences in collateral perfusion between groups probably did not account for the observed results, but coronary collateral blood flow was not specifically quantified. The reductions in myocardial necrosis produced by helium in the absence or presence of other drug interventions occurred independent of changes in major determinants of myocardial oxygen consumption. Nevertheless, coronary venous oxygen tension was not directly measured nor was myocardial oxygen consumption calculated. Notably, no significant differences in hemodynamics were observed amongst groups before and during coronary artery occlusion that may account for differences in infarct size observed between groups. Finally, the current results implicating a role for ROS and mitochondrial $\mathrm{K}_{\mathrm{ATP}}$ channels in heliuminduced cardioprotection were obtained in barbiturate-anesthetized, acutely instrumented rabbits. Whether similar results occur in other animal species or humans is unknown. Helium preconditioning has yet to be established in humans, but administration of this noble gas before a defined of period of myocardial ischemia be beneficial in a clinical setting in which an anesthetic is not required (e.g., inflation of an angioplasty balloon during cardiac catheterization). However, additional investigation will be required to test this intriguing hypothesis.

In summary, the current results confirm that brief, intermittent administration of helium before prolonged coronary artery occlusion and reperfusion protects myocardium against infarction. 
The findings further suggest that ROS and mitochondrial $\mathrm{K}_{\mathrm{ATP}}$ channels mediate this heliuminduced preconditioning in vivo.

\section{Acknowledgements}

This work was supported in part by National Institutes of Health grants HL 054820 and GM 066730 from the United States Public Health Service (Bethesda, MD) and by departmental funds. Dr. Amour is the recipient of research fellowship grants from the Société Française d'Anesthésie et de Réanimation (SFAR, Paris, France), Novo Nordisk® (Paris-La Défense, France), and the Assistance Publique des Hôpitaux de Paris (APHP, Paris, France).

\section{References}

1. Zweier JL, Flaherty JT, Weisfeldt ML. Direct measurement of free radical generation following reperfusion of ischemic myocardium. Proc Natl Acad Sci USA 1987;84:1404-1407. [PubMed: 3029779]

2. Ambrosio G, Zweier JL, Duilio C, et al. Evidence that mitochondrial respiration is a source of potentially toxic oxygen free radicals in intact rabbit hearts subjected to ischemia and reflow. J Biol Chem 1993;268:18532-18541. [PubMed: 8395507]

3. Baines CP, Goto M, Downey JM. Oxygen radicals released during ischemic preconditioning contribute to cardioprotection in the rabbit myocardium. J Mol Cell Cardiol 1997;29:207-216. [PubMed: 9040035]

4. Vanden Hoek TL, Becker LB, Shao Z, et al. Reactive oxygen species released from mitochondria during brief hypoxia induce preconditioning in cardiomyocytes. J Biol Chem 1998;273:18092-18098. [PubMed: 9660766]

5. Tanaka M, Fujiwara H, Yamasaki K, et al. Superoxide dismutase and N-2-mercaptopropionyl glycine attenuate infarct size limitation effect of ischaemic preconditioning in the rabbit. Cardiovasc Res 1994;28:980-986. [PubMed: 7954610]

6. Mullenheim J, Ebel D, Frassdorf J, et al. Isoflurane preconditions myocardium against infarction via release of free radicals. Anesthesiology 2002;96:934-940. [PubMed: 11964602]

7. Tanaka K, Weihrauch D, Kehl F, et al. Mechanism of preconditioning by isoflurane in rabbits: a direct role for reactive oxygen species. Anesthesiology 2002;97:1485-1490. [PubMed: 12459675]

8. Ludwig LM, Tanaka K, Eells JT, et al. Isoflurane-induced preconditioning is mediated by reactive oxygen species generated by mitochondrial electron transport chain complex III. Anesth Analg 2004;99:1308-1315. [PubMed: 15502022]

9. Kohro S, Hogan QH, Nakae Y, et al. Anesthetic effects on mitochondrial ATP-sensitive K channel. Anesthesiology 2001;95:1435-1340. [PubMed: 11748403]

10. Tanaka K, Weihrauch D, Ludwig LM, et al. Mitochondrial adenosine triphosphate-regulated potassium channel opening acts as a trigger for isoflurane-induced preconditioning by generating reactive oxygen species. Anesthesiology 2003;98:935-943. [PubMed: 12657856]

11. Pagel PS, Krolikowski JG, Venkatapuram S, et al. Noble gases without anesthetic properties protect myocardium against infarction by activating prosurvival signaling kinases and inhibiting mitochondrial permeability transition in vivo. Anesth Analg 2007;105:562-569. [PubMed: 17717207]

12. Pagel PS, Krolikowski JG, Weihrauch D, et al. Inhibition of glycogen synthase kinase or the apoptotic protein p53 lowers the threshold of helium cardioprotection in vivo: role of mitochondrial permeability transition. Anesth Analg 2008;107(In Press)

13. Pagel PS, Krolikowski JG, Pratt PF Jr, et al. Mechanism of helium-induced preconditioning: a direct role for nitric oxide in rabbits. Anesth Analg 2008;106(In Press)

14. Weber NC, Toma O, Damla H, et al. Upstream signaling of PKC-e in xenon-induced pharmacological preconditioning. Implication of mitochondrial $\mathrm{K}_{\mathrm{ATP}}$ channels and PDK-1. Eur J Pharmacol 2006;539:1-9. [PubMed: 16716295]

15. Warltier DC, Zyvoloski MG, Gross GJ, et al. Determination of experimental myocardial infarct size. J Pharmacol Methods 1981;6:199-210. [PubMed: 7329069]

16. Wallenstein S, Zucker CL, Fleiss JL. Some statistical methods useful in circulation research. Circ Res 1980;47:1-9. [PubMed: 7379260] 
17. Koblin DD, Fang Z, Eger EI II, et al. Minimum alveolar concentrations of noble gases, nitrogen, and sulfur hexafluoride in rats: helium and neon as nonimmobilizers (nonanesthetics). Anesth Analg 1998;87:419-424. [PubMed: 9706943]

18. Tritto I, D'Andrea D, Eramo N, et al. Oxygen radicals can induce preconditioning in rabbit hearts. Circ Res 1997;80:743-748. [PubMed: 9130455]

19. Pain T, Yang XM, Critz SD, et al. Opening of mitochondrial $\mathrm{K}_{\mathrm{ATP}}$ channels triggers the preconditioned state by generating free radicals. Circ Res 2000;87:460-466. [PubMed: 10988237]

20. Carroll R, Grant VA, Yellon DM. Mitochondrial K(ATP) channel opening protects a human atrialderived cell line by a mechanism involving free radical generation. Cardiovasc Res 2001;51:691700. [PubMed: 11530102]

21. Forbes RA, Steenbergen C, Murphy E. Diazoxide-induced cardioprotection requires signaling through a redox-sensitive mechanism. Circ Res 2001;88:802-809. [PubMed: 11325872]

22. Obata T, Yamanaka Y. Block of cardiac ATP-sensitive $\mathrm{K}(+)$ channels reduces hydroxyl radicals in the rat myocardium. Arch Biochem Biophys 2000;378:195-200. [PubMed: 10860536]

23. Novalija E, Varadarajan SG, Camara AK, et al. Anesthetic preconditioning: triggering role of reactive oxygen and nitrogen species in isolated hearts. Am J Physiol Heart Circ Physiol 2002;283:H44-H52. [PubMed: 12063273]

24. Kevin LG, Novalija E, Riess ML, et al. Sevoflurane exposure generates superoxide but leads to decreased superoxide during ischemia and reperfusion in isolated hearts. Anesth Analg 2003;96:949955. [PubMed: 12651639]

25. Aruoma OI, Halliwell B, Hoey BM, et al. The antioxidant action of N-acetylcysteine: its reaction with hydrogen peroxide, hydroxyl radical, superoxide, and hypochlorous acid. Free Radic Biol Med 1989;6:593-597. [PubMed: 2546864]

26. Beyersdorf F, Fuchs J, Eberhardt B, et al. Myocardial protection by 2-mercaptopropionylglycine during global ischemia in dogs. Arzneimittelforschung 1989;39:46-49. [PubMed: 2470381]

27. Beyersdorf F, Zimmer G, Fuchs J, et al. Improvement of myocardial function after global hypoxia by protection of the inner mitochondrial membrane. Arzneimittelforschung 1987;37:142-149. [PubMed: 2953343]

28. Fuchs J, Mainka L, Zimmer G. 2-mercaptopropionylglycine and related compounds in treatment of mitochondrial dysfunction and postischemic myocardial damage. Arzneimittelforschung 1985;35:1394-1402. [PubMed: 2935161]

29. Maxwell MP, Hearse DJ, Yellon DM. Species variation in the coronary collateral circulation during regional myocardial ischaemia: a critical determinant of the rate of evolution and extent of myocardial infarction. Cardiovasc Res 1987;21:737-746. [PubMed: 3440266]

J Cardiothorac Vasc Anesth. Author manuscript; available in PMC 2009 August 1. 
CONTROL

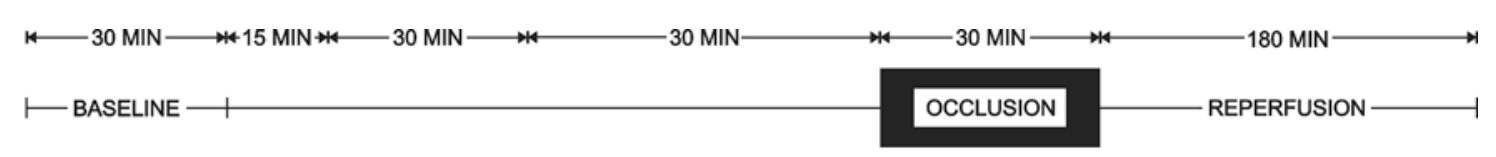

HELIUM

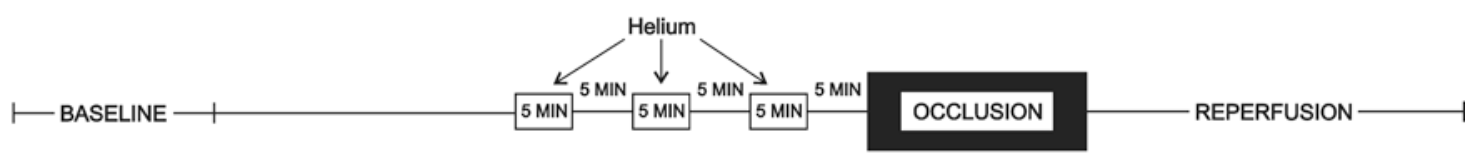

NAC
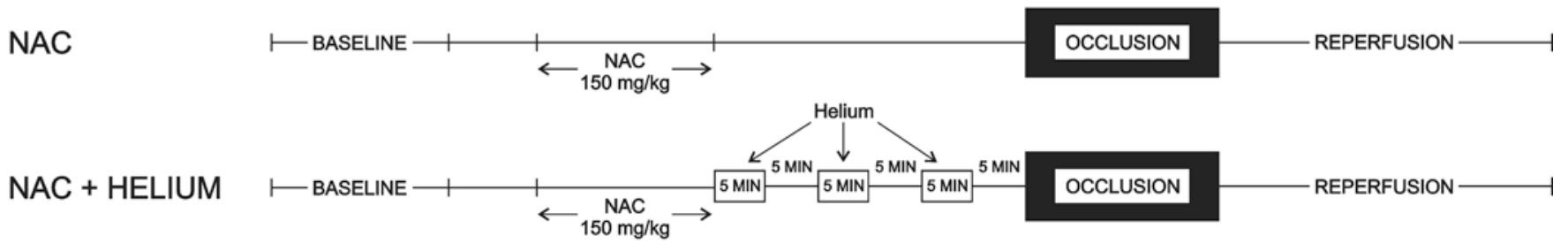

2-MPG

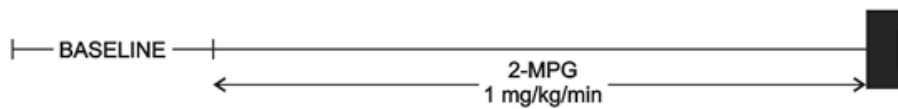

OCCLUSION REPERFUSION

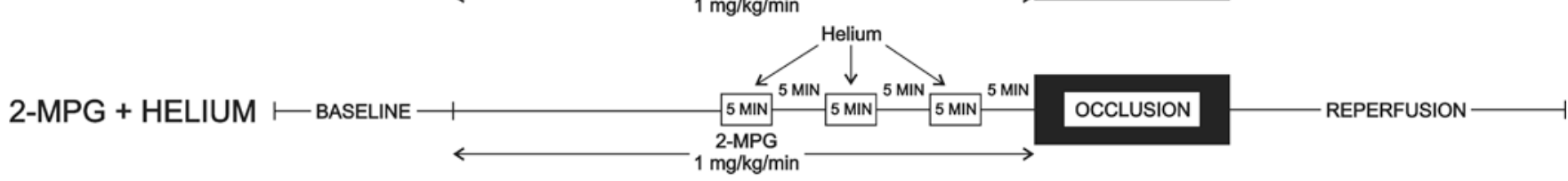

5-HD
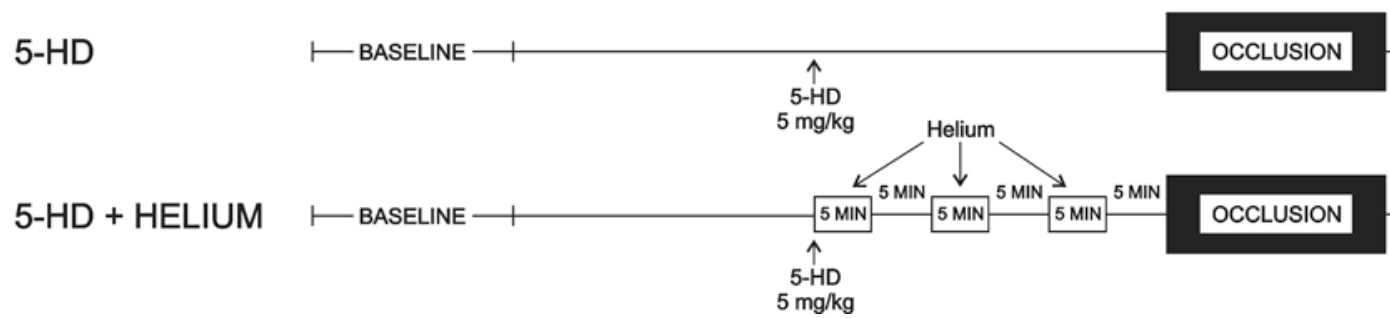

OCCLUSION REPERFUSION

Figure 1.

Schematic illustration depicting the experimental protocol used in the current investigation. Abbreviations: $\mathrm{NAC}=\mathrm{N}$-acetylcysteine; $2-\mathrm{MPG}=\mathrm{N}$-2-mercaptoproprionyl glycine; 5 - $\mathrm{HD}=$ 5-hydroxydecanoate 


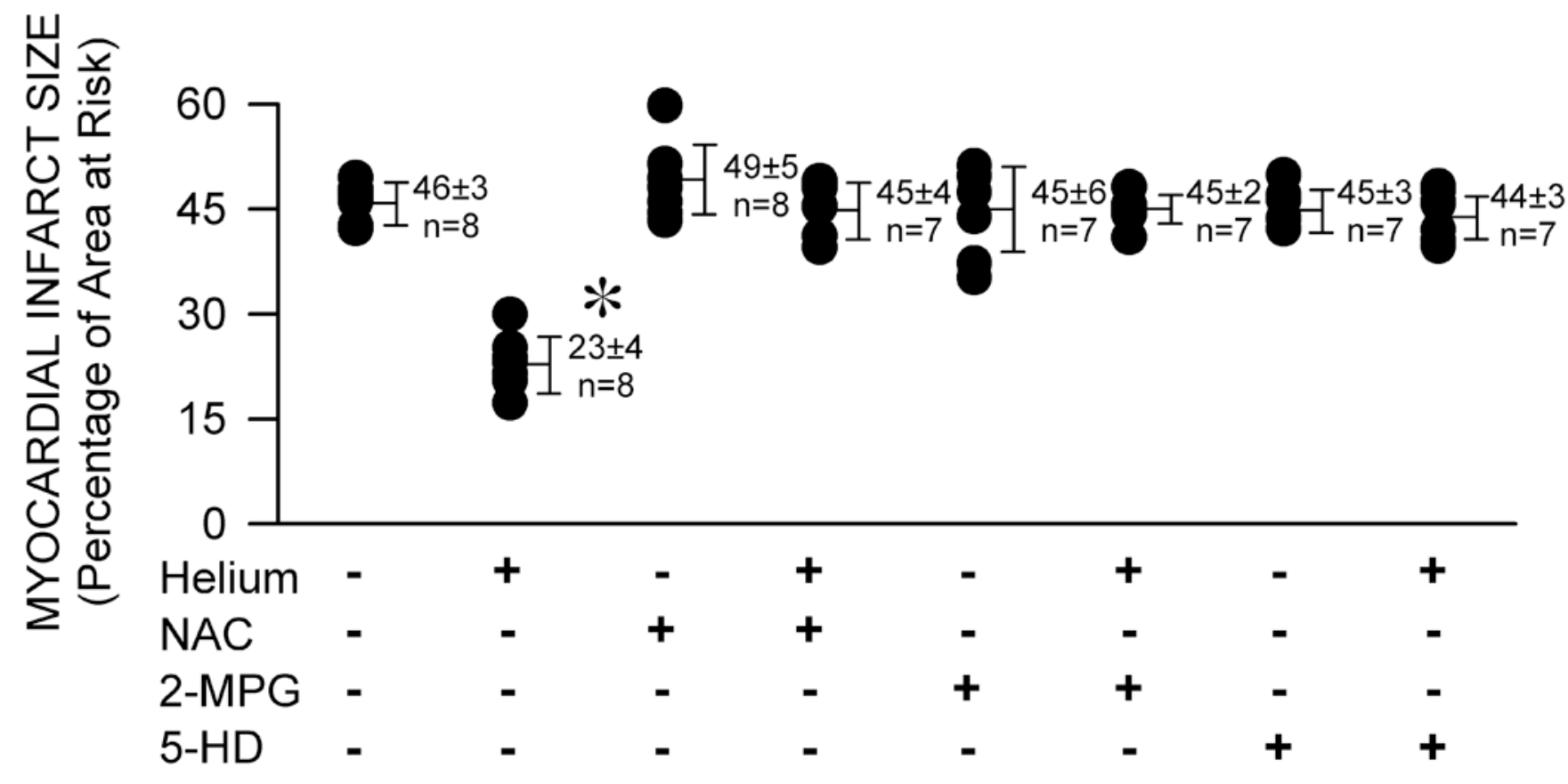

Figure 2.

Myocardial infarct size depicted as a percentage of left ventricular area at risk in rabbits receiving $0.9 \%$ saline (control, $\mathrm{CON}$ ) or three cycles of $70 \%$ helium-30\% oxygen administered for 5 min interspersed with 5 min of an air-oxygen mixture $\left(\mathrm{F}_{\mathrm{i}} \mathrm{O}_{2}=0.30\right)$ in the presence or absence of pretreatment with the ROS scavengers $N$-acetylcysteine (NAC; $150 \mathrm{mg} / \mathrm{kg}$ ) or $\mathrm{N}$-2-mercaptoproprionyl glycine (2-MPG; $75 \mathrm{mg} / \mathrm{kg}$ ), or the selective mitochondrial $\mathrm{K}_{\mathrm{ATP}}$ channel antagonist 5 -hydroxydeconate $(5-\mathrm{HD} ; 5 \mathrm{mg} / \mathrm{kg}$ ) before prolonged coronary artery occlusion and reperfusion. Each point represents a single experiment. All data are mean \pm SD. *Significantly $(\mathrm{P}<0.05)$ different from $\mathrm{CON}$ 


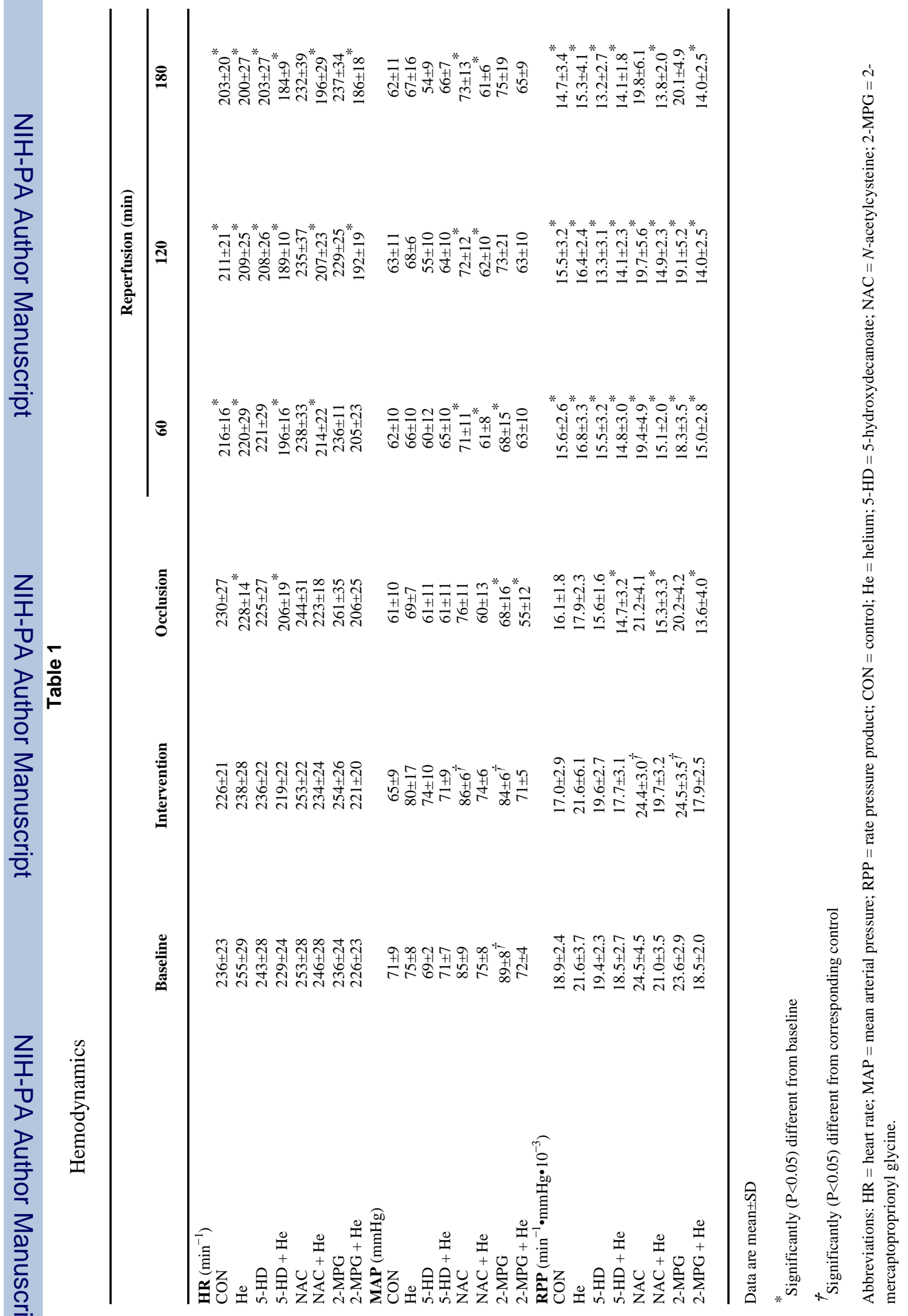




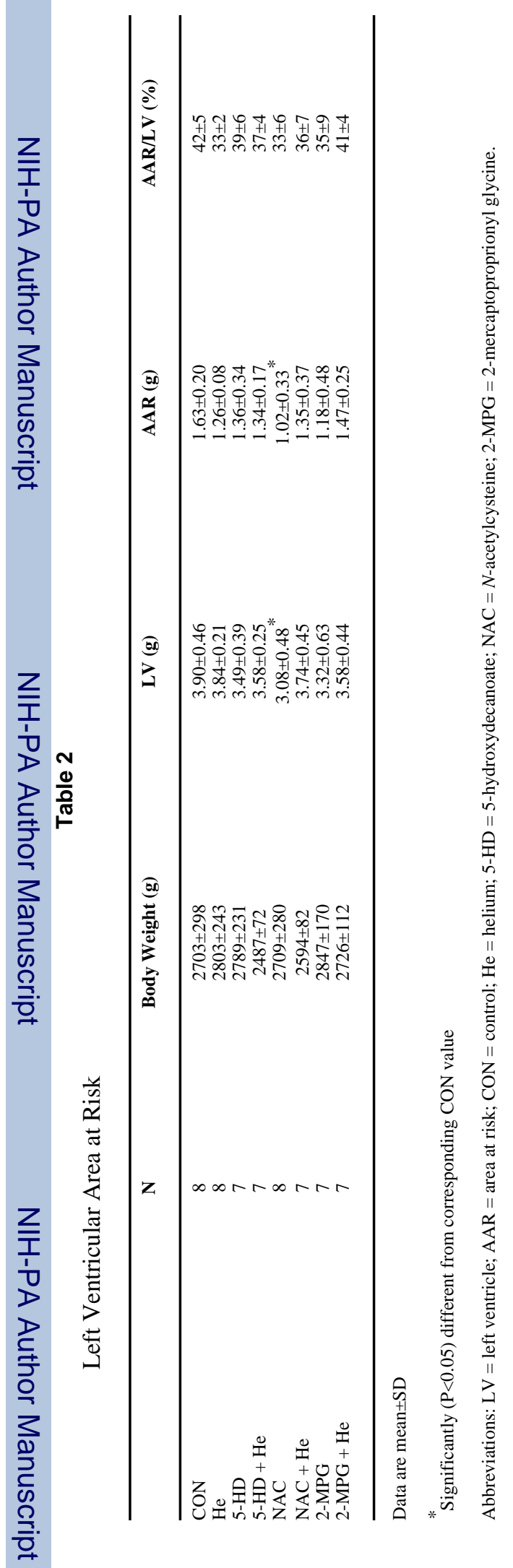

